\title{
Microstructures and Soft Magnetic Properties of High Saturation Magnetization Fe-Co-N alloy Thin Films
}

\author{
N. X. Sun and S. X. Wang \\ Department of Material Sciences and Engineering, Stanford University \\ Stanford, CA 94305-4045, USA \\ Chin-Ya Hung, Chester X. Chien and Hua-Ching Tong \\ Read-Rite Corporation, 44100 Osgood Road, Fremont, CA 94539
}

\begin{abstract}
High saturation magnetization soft magnetic materials are required for future high-density recording heads as well as high frequency inductors. In this work, $\left(\mathrm{Fe}_{0.7} \mathrm{Co}_{0.3}\right)_{1-x} \mathrm{~N}_{x}$ (or in short $\mathrm{FeCoN}$ ) alloy films were synthesized with a high saturation magnetization of $24.5 \mathrm{kG}$, a hard axis coercivity of $5 \mathrm{Oe}$, an easy axis coercivity of $18 \mathrm{Oe}$, and a resistivity of $55 \mu \Omega \cdot \mathrm{cm}$. The FeCoN film sandwiched between two permalloy layers $(5 \mathrm{~nm})$ shows very good magnetic softness, a low hard axis coercivity of $0.6 \mathrm{Oe}$, an easy axis coercivity of $7.8 \mathrm{Oe}$, an excellent inplane uniaxial anisotropy with an anisotropy of about 20 Oe, an initial permeability of 1000 , and a roll-off frequency of $1.5 \mathrm{GHz}$. In order to understand the effect of the permalloy layers on the FeCoN layer, we fabricated four film structures: single layer $\mathrm{FeCoN}$ film; $\mathrm{FeCoN}$ film sandwiched between two permalloy layers on both sides; FeCoN film with one permalloy layer as the underlayer; and $\mathrm{FeCoN}$ film with one permalloy layer as caplayer. All these film structures were both magnetically and structurally characterized and compared. Structural characterization shows that there is no significant difference in the grain size of the $\mathrm{FeCoN}$ single layer and the FeCoN layer sandwiched between two permalloy layers. The four film structures have almost the same amount of compressive stress, about $-300 \mathrm{MPa}$; and their saturation magnetostriction constants are also very close, in the range of $39.6 \times 10^{-6}$ to $44.3 \times 10^{-6}$. Difference in the crystallographic textures was observed in the pole figures for the $\mathrm{FeCoN}$ single layer and $\mathrm{FeCoN}$ film with permalloy underlayer.
\end{abstract}

\section{INTRODUCTION}

Soft magnetic thin films with high saturation magnetization are highly desired in a wide range of applications from magnetic write heads to high frequency inductors. However, soft magnetic thin-film materials with a saturation magnetization of $>21 \mathrm{kG}$ and a low coercivity of less than $1 \mathrm{Oe}$ are still not available, although magnetic materials with saturation magnetization higher than $21 \mathrm{kG}$ are readily obtainable in the binary $\mathrm{Fe}_{1-x} \mathrm{Co}_{x}$ alloys [1]. Discovering new soft magnetic materials with very high saturation magnetization remains to be a major challenge.

Magnetic softness of polycrystalline thin films is an extrinsic property of the magnetic materials, and is closely related to the structural and compositional characteristics in the thin films, such as grain size [2], crystallographic texture [3], strain and stress state [4]. These characteristics can also affect anisotropy and magnetostriction, which are in turn correlated with the magnetic softness. However, it is still not well established how these structural characteristics affect the magnetic softness. 
In the last decade, huge amount of research was carried out on the soft magnetic FeMN alloy thin films, with the typical M being Al [5], Ta [6], Cr [7], etc., and the M composition being in the range of $0 \sim 10$ at $\%$. Enormous efforts are being spent on optimizing the microstructure and composition of these FeMN thin films materials, trying to get optimal soft magnetic properties. These FeMN thin films have a typical saturation magnetization of 18 to $20 \mathrm{kG}$, a coercivity of 1 Oe, an electrical resistivity of $\sim 40 \mu \Omega \cdot \mathrm{cm}$, and an anisotropy field of $5 \sim 10$ Oe [2-4]. However, the saturation magnetization of these FeMN thin films is apparently limited by that of pure iron which is $21.5 \mathrm{kG}$ [1].

It is well known that the binary Fe-Co alloys have a very high saturation magnetization of $24.5 \mathrm{kG}$ in the composition range of $\mathrm{Fe}_{1-x} \mathrm{Co}_{x}(0.3<x<0.4)$ [1]. It would be of great scientific as well as technological interests to make this alloy soft while keeping its high saturation magnetization at the same time. However, the extremely high saturation magnetostriction in the Fe-Co alloys, being about $40 \sim 65 \times 10^{-6}$ in the composition range of $30 \sim 40$ at $\%$ of cobalt, makes it very difficult to achieve low coercivity or in-plane uniaxial anisotropy [8-10].

In this work we successfully fabricated the Fe-Co alloy based films soft, while keeping the high saturation magnetization by introducing an appropriate amount of $\mathrm{N}$ into the binary Fe-Co alloys first, and then sandwiching the obtained FeCoN films with two very thin layers of permalloy film. The various magnetic and structural characteristics have been studied to understand how the structural characteristics are related to the soft magnetic properties of the FeCoN films.

\section{EXPERIMENT}

FeCoN films were deposited onto (100) Si wafer through reactive RF diode sputtering in an argon and nitrogen atmosphere. The target composition was $\mathrm{Fe}_{70} \mathrm{Co}_{30}$ (at\%) with a purity of $99.95 \%$. The base pressure of the sputtering chamber was $\sim 2 \times 10^{-7}$ Torr. The gas flow rate of argon was set constant; while the flow rate of nitrogen was adjusted to get samples with different $\mathrm{N}$ contents. Permalloy layer was deposited in a pure argon atmosphere by RF sputtering. A magnetic field of about 50 Oe was applied during deposition.

For the saturation magnetization measurements, FeCoN films with different $\mathrm{N}$ contents were deposited onto Si substrates with an area of $10 \times 10 \mathrm{~mm}^{2}$. FeCoN Film thickness, ranging from 0.8 to $1.2 \mu \mathrm{m}$ for all saturation magnetization measurements, is measured by both a Dektak II Surface Profilometer and a Tencor Alpha-step Profiler for each deposition batch. Magnetic properties of the obtained films were characterized by a vibrating sample magnetometer (VSM). The magnetic moment was carefully calibrated by a nickel film standard with a purity of $99.999 \%$ and a film volume of $10 \times 10 \times 0.002 \mathrm{~mm}^{3}$. The moment calibration was done at a field of 5000 Oe and a temperature of $\sim 20{ }^{\circ} \mathrm{C}$. The hard-axis magnetic permeability was measured by a custom-built permeameter with a frequency range up to $3 \mathrm{GHz}$ on samples with an area of $5 \times 5$ $\mathrm{mm}^{2}$. The details of the permeameter are available in reference [11]. The resistivities of these FeCoN films were measured with a four-point probe station. The compositions of the FeCoN films were analyzed by X-ray photoelectron spectroscopy (XPS). A Philips transmission electron microscope (CM20 FEG-TEM) operated at a voltage of $200 \mathrm{kV}$ was used for microstructure analysis.

A four-circle diffractometer operated at $45 \mathrm{kV} / 30 \mathrm{~mA}$ was utilized for pole figure analysis. In this diffractometer, three rotations, namely, $\phi, \chi$ and $\omega$ change specimen orientation relative to the incident X-ray beam; the fourth rotation, $2 \theta$, positions the detector relative to the incident 
beam. The pole figures were generated by doing the $\phi$ scans at different $\chi$ angles at a fixed $2 \theta$ value, which corresponds to a specific Bragg peak of the thin films. In this analysis, we fixed the $2 \theta$ at the (110) Bragg peak of the $\mathrm{BCC} \mathrm{Fe}(\mathrm{Co}, \mathrm{N})$ phase. The range of $\phi$ and $\chi$ are $0 \sim 360^{\circ}$ and 0 $\sim 90^{\circ}$, respectively, with a step of $1^{\circ}$ for both $\phi$ and $\chi$. The pole figures were shown in a stereographic projection.

The film stress was measured by using a wafer-curvature method. The wafer curvature measurement relies on the fact that when a thin film is under stress, it imposes a bending moment on the substrate. The curvature change of the substrate before and after deposition can be related to the biaxial stress of the thin film by [12]:

$$
\sigma_{f}=\frac{M_{s} t_{s}^{2}}{6 t_{f}} \Delta K,
$$

where $\sigma_{f}$ is the biaxial stress, $t_{s}$ is the thickness of the substrate, $t_{f}$ is the thickness of the film, $M_{s}$ is the biaxial modulus of the substrate, and $\Delta K$ is the curvature change of the substrate before and after film deposition. The curvature is obtained by scanning a laser beam across the wafer and measuring the deflection of the reflected beam. By assuming that the film is much thinner than the substrate, the biaxial modulus can be expressed by: $M_{s}=E_{s} /\left(1-v_{s}\right)$, where $E_{\mathrm{s}}$ and $v_{s}$ are the Young's modulus and the Poission ratio of Si substrate, respectively. Here, $M_{s}=200 \mathrm{GPa}$ is used for the (100) Si wafer. Standard mirrors with known radius of curvature of 20, 30, 60 meters, and infinity (flat mirror) were used for calibration.

Saturation Magnetostriction constant of these FeCoN thin films was measured with an automated magnetostriction tester manufactured by Lafouda Solution. This is a direct method of measuring saturation magnetostriction constant with a high precision of $<10^{-8}$. During the measurement, an applied in-plane rotating magnetic field changes the magnetization direction of the film, and the magnetic thin film on Si substrate beam will bend due to the length change of the magnetic thin film. The magnitude of the rotational field is 50 Oe. A laser beam shining on the film is utilized to measure the small bending of the film and substrate. The deflection angle of the bending beam at the measuring point, $\phi$, can be expressed as [13]:

$$
\phi=\frac{6 l \varepsilon}{t_{s}^{2}} \times\left(t_{f} \lambda\right),
$$

where $t_{f}$ and $t_{s}$ are the thickness of the film and substrate, respectively, $l$ is the distance between the clamping edge to the measuring point, $\lambda$ is the magnetostriction constant at the applied field, and $\varepsilon=E_{f}\left(1-v_{s}\right) / E_{s}\left(1+v_{f}\right)$, where $E_{f}, E_{s}$ and $v_{s}, v_{f}$ are the Young's modulus and Poisson's ratio of the film and substrate, respectively. Here, $\varepsilon=0.71$ is used for all measurements.

\section{RESULTS AND DISCUSSION}

\section{MAGNETIC PROPERTIES}

The saturation magnetization $\left(4 \pi M_{s}\right)$ of the as-deposited FeCoN films is shown in Fig. 1 as a function of the $\mathrm{N}_{2} / \mathrm{Ar}$ gas flow rate ratio. It is clear that the saturation magnetization values 
scatters between $25 \pm 1 \mathrm{kG}$ in a wide range of $\mathrm{N}_{2} / \mathrm{Ar}$ flow rate ratio of $0 \% \sim 7 \%$, indicating that the saturation magnetization of the FeCoN films are almost identical to that of the pure $\mathrm{Fe}_{70} \mathrm{Co}_{30}$ alloy film. Since the widely accepted highest moment for the FeCo binary alloy is $24.5 \mathrm{kG}$ in the composition range of $\mathrm{Fe}_{1-x} \mathrm{Co}_{x}(0.3<x<0.4)$ [1], the measured saturation magnetization for the FeCo alloy film, of which the composition was determined by XPS to be around $\mathrm{Fe}_{67} \mathrm{Co}_{33}$, is conservatively taken as $24.5 \mathrm{kG}$. Therefore, the FeCoN films at $\mathrm{N}_{2} / \mathrm{Ar}$ flow rate ratios less than $7 \%$ should also have a high saturation magnetization of $24.5 \mathrm{kG}$. At higher $\mathrm{N}_{2} / \mathrm{Ar}$ flow rate ratios, the saturation magnetization values of the FeCoN alloys drop gradually. Admittedly, one of our initial purposes of choosing the FeCoN alloy system was to find out whether we could possibly find the allusive high moment $\left(\mathrm{Fe}_{1-\mathrm{x}} \mathrm{Co}_{\mathrm{x}}\right)_{16} \mathrm{~N}_{2}$ phase. Unfortunately, we did not find any sign of this phase in the as deposited FeCoN films.
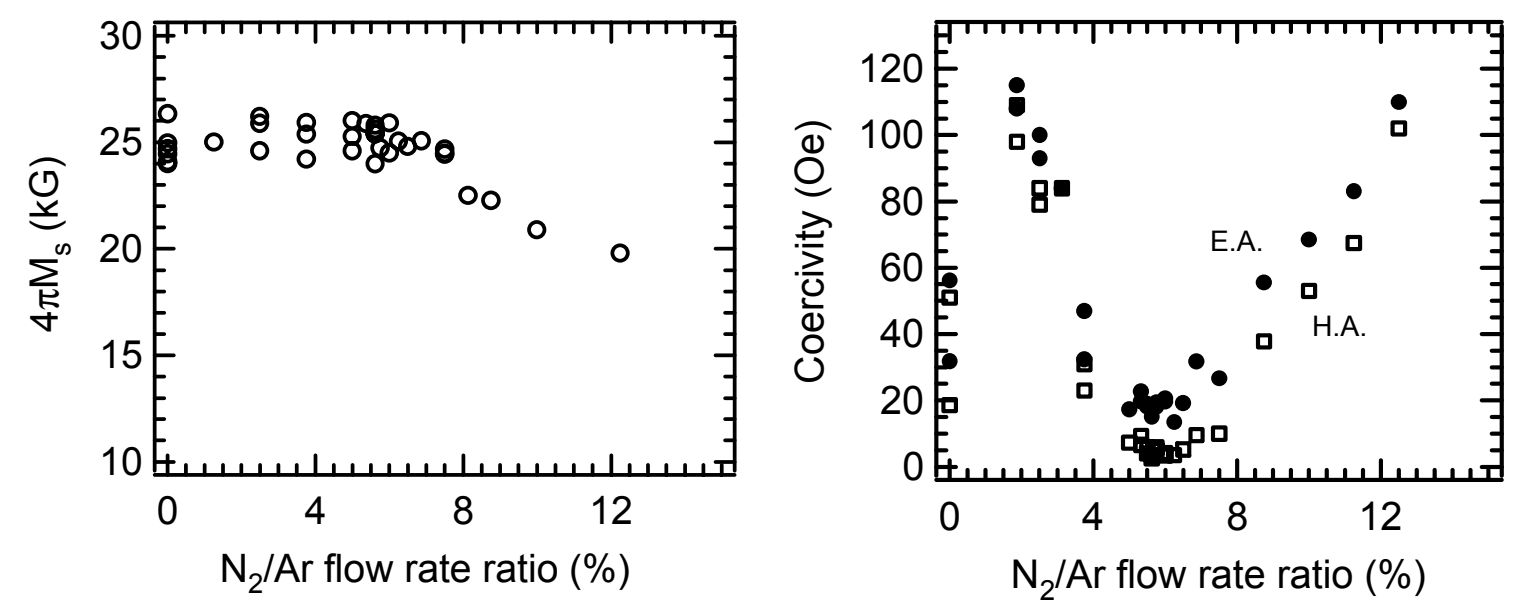

Figure 1: $4 \pi M_{S}$ vs. $N_{2} / A r$ gas flow rate ratio

Figure 2: Hard axis (H.A.) and easy axis (H.A.) coercivity vs. $\mathrm{N}_{2} /$ Ar flow rate ratio for $\mathrm{FeCoN}$ films.

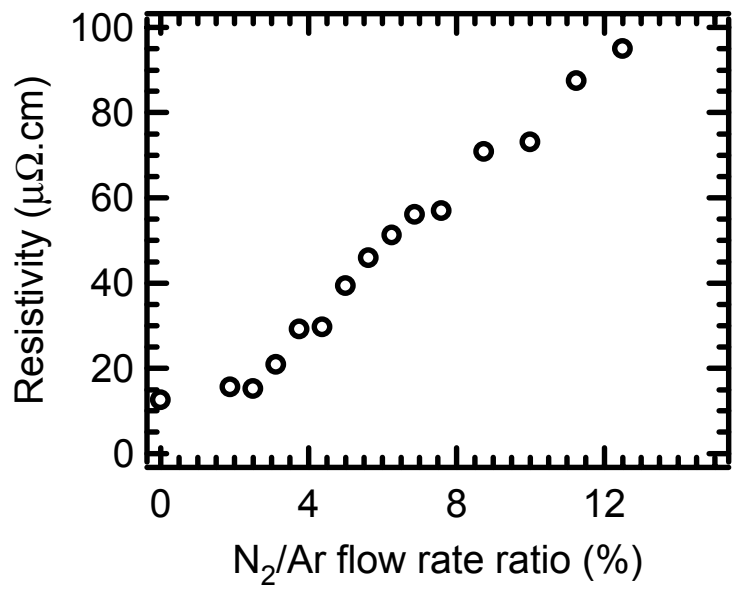

Figure 3: Resistivity of FeCoN films versus $\mathrm{N}_{2} / \mathrm{Ar}$ gas flow rate ratios. 
The coercivity $\left(H_{c}\right)$ of these FeCoN films is shown in Fig. 2 as a function of the $\mathrm{N}_{2} / \mathrm{Ar}$ gas flow rate ratio. The hard-axis coercivity first decreases quickly from about $100 \mathrm{Oe}$ at a gas flow rate ratio of $2 \%$ to around $5 \mathrm{Oe}$ at a $\mathrm{N}_{2} / \mathrm{Ar}$ gas flow rate ratio of $5 \sim 6 \%$, then the coercivity increases with the increment of the gas flow rate ratio. The easy axis coercivity has the same trend as the hard axis coercivity, but it is higher than that of the hard axis, with a minimum of about 20 Oe. Nitrogen content in the FeCoN films was determined by XPS to be about 5 at $\%$, and the $\mathrm{Fe} / \mathrm{Co}$ atomic ratio is $2 / 1$ at the $\mathrm{N}_{2} / \mathrm{Ar}$ gas flow rate ratio of $5.6 \%$. At different $\mathrm{N}_{2} / \mathrm{Ar}$ gas flow rate ratios, the $\mathrm{Fe} / \mathrm{Co}$ atomic ratio is almost constant, being within $(2 \pm 0.1) / 1$.

The resistivity increases as the increment of the $\mathrm{N}_{2} / \mathrm{Ar}$ gas flow rate ratio, as shown in Fig. 3. For the FeCo films, the resistivity is around $12 \mu \Omega \cdot \mathrm{cm}$; while the resistivity increases almost linearly with the gas flow rate, reaching $55 \mu \Omega \cdot \mathrm{cm}$ at a $\mathrm{N}_{2} / \mathrm{Ar}$ gas flow rate ratio of $5.6 \%$, where the lowest coercivity is obtained.

Combining Figs. 1 to 3, we can see that the high saturation magnetization of around $24.5 \mathrm{kG}$ and low coercivity of around 5 Oe can be realized at a $\mathrm{N}_{2} / \mathrm{Ar}$ gas flow rate ratio of $5 \sim 6 \%$. However, the FeCoN single layer is not magnetically soft enough for such applications as write head materials. Making artificial magnetic structures have been proven to be an effective way to fabricate magnetically soft thin films. For example, laminating magnetic thin films with dielectric thin layers leads to much better magnetic softness [14]. For the purpose of lowering the coercivity further, four magnetic structures were fabricated and their magnetic properties compared. The schematic graphs of the four film structures are indicated in Figure 4, they are the FeCoN single layer (in short: $\mathrm{FeCoN}$ ), the $\mathrm{FeCoN}$ layer with a very thin permalloy underlayer $(\mathrm{P} \backslash \mathrm{FeCoN})$, the $\mathrm{FeCoN}$ layer sandwiched into two thin permalloy layers $(\mathrm{P} \backslash \mathrm{FeCoN} \backslash \mathrm{P})$, and the FeCoN layer with a permalloy caplayer $(\mathrm{FeCoN} \backslash \mathrm{P})$. All the permalloy layers in these four structures are $5 \mathrm{~nm}$ thick, and all the FeCoN layer thickness is $100 \mathrm{~nm}$.

FeCoN layer, $100 \mathrm{~nm}$

a

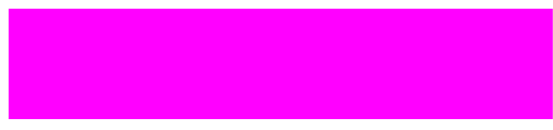

c

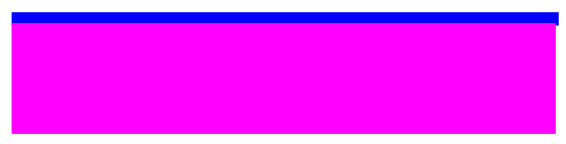

FeCoN layer, $100 \mathrm{~nm}$

b

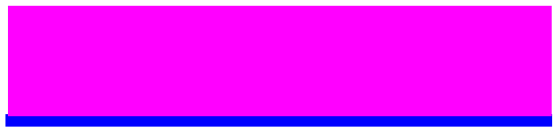

Permalloy layer, $5 \mathrm{~nm}$

d

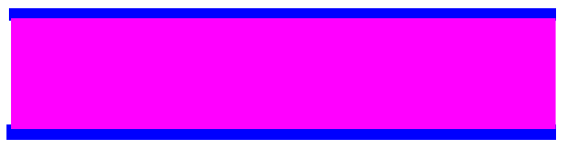

Figure 4: Schematic of the four film structures, a: FeCoN; b: P|FeCoN; c: FeCoN|P; d: P|FeCoN|P.

The hysteresis loops of the four film structures are shown in Figs. 5(a) to 5(d). The coercivity of the $\mathrm{FeCoN}$ single layer is about 5 and 18 Oe in the hard and easy axis, respectively. The hard and easy axis coercivity of the $\mathrm{P} \backslash \mathrm{FeCoN}$ film structure drops to 2 Oe and $10 \mathrm{Oe}$, respectively. Also, hard axis loop becomes more linear, indicating that the $\mathrm{P} \backslash \mathrm{FeCoN}$ structure has a better 
uniaxial anisotropy than the FeCoN single layer. What is striking is that for the sandwiched $\mathrm{P} \backslash \mathrm{FeCoN} \backslash \mathrm{P}$ film structure, the hard and easy axis coercivity is further reduced to 0.6 and $7.8 \mathrm{Oe}$, respectively. Furthermore, the square easy axis loop, and an almost linear hard axis loop of the sandwiched $\mathrm{FeCoN}$ film indicate an excellent in-plane uniaxial anisotropy. The anisotropy field is determined to be 20 Oe. However, the FeCoN $\backslash P$ structure has similar hysterisis loops as the FeCoN single layer.
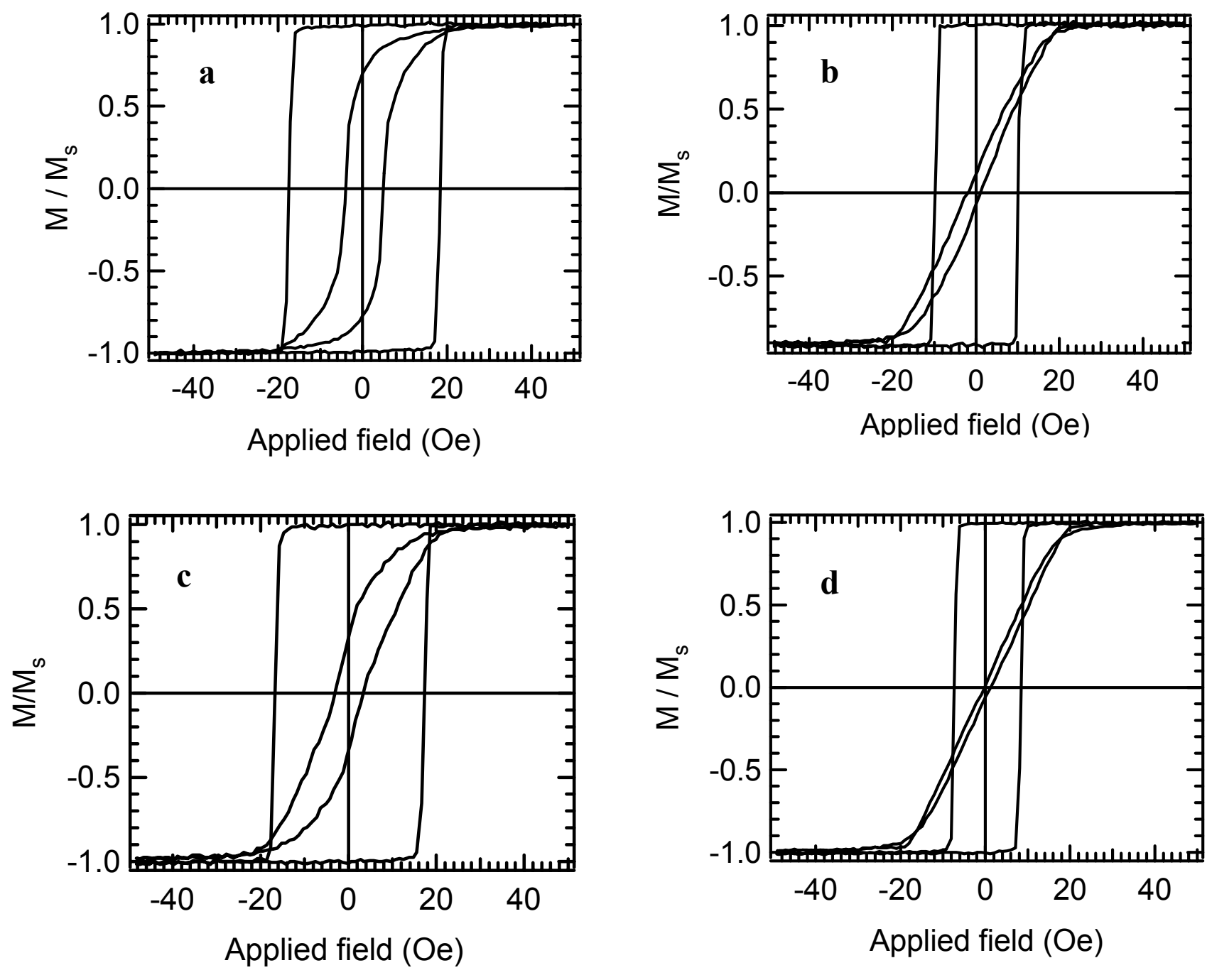

Figure 5 Typical easy axis loops (square loops) and hard axis loops (slender loops) for the four film structures shown in Figure 4, a: FeCoN; b: P|FeCoN; c: FeCoN $\backslash P$; d: P|FeCoN $\mid P$.

High frequency permeability measurement shows the P\FeCoN\P film structure has an initial relative permeability of 1000 , and a $3 \mathrm{~dB}$ roll-off frequency of $1.5 \mathrm{GHz}$ [15]. The combination of the high initial permeability and high roll-off frequency makes this film structure a very promising candidate for applications in the future high frequency inductors and inductive 
write heads. The saturation magnetization of representative soft magnetic materials versus the year when they were reportedly incorporated into magnetic recording heads (except the $\mathrm{FeCoN}$ in this work) is shown in Fig. 6 as a comparison. It is very clear that the saturation magnetization for the soft magnetic materials incorporated for write heads are increasing steadly, and that the saturation magnetization for the $\mathrm{FeCoN}$ is much higher than $20 \mathrm{kG}$, the widely accepted saturation magnetization limit for the write head materials.

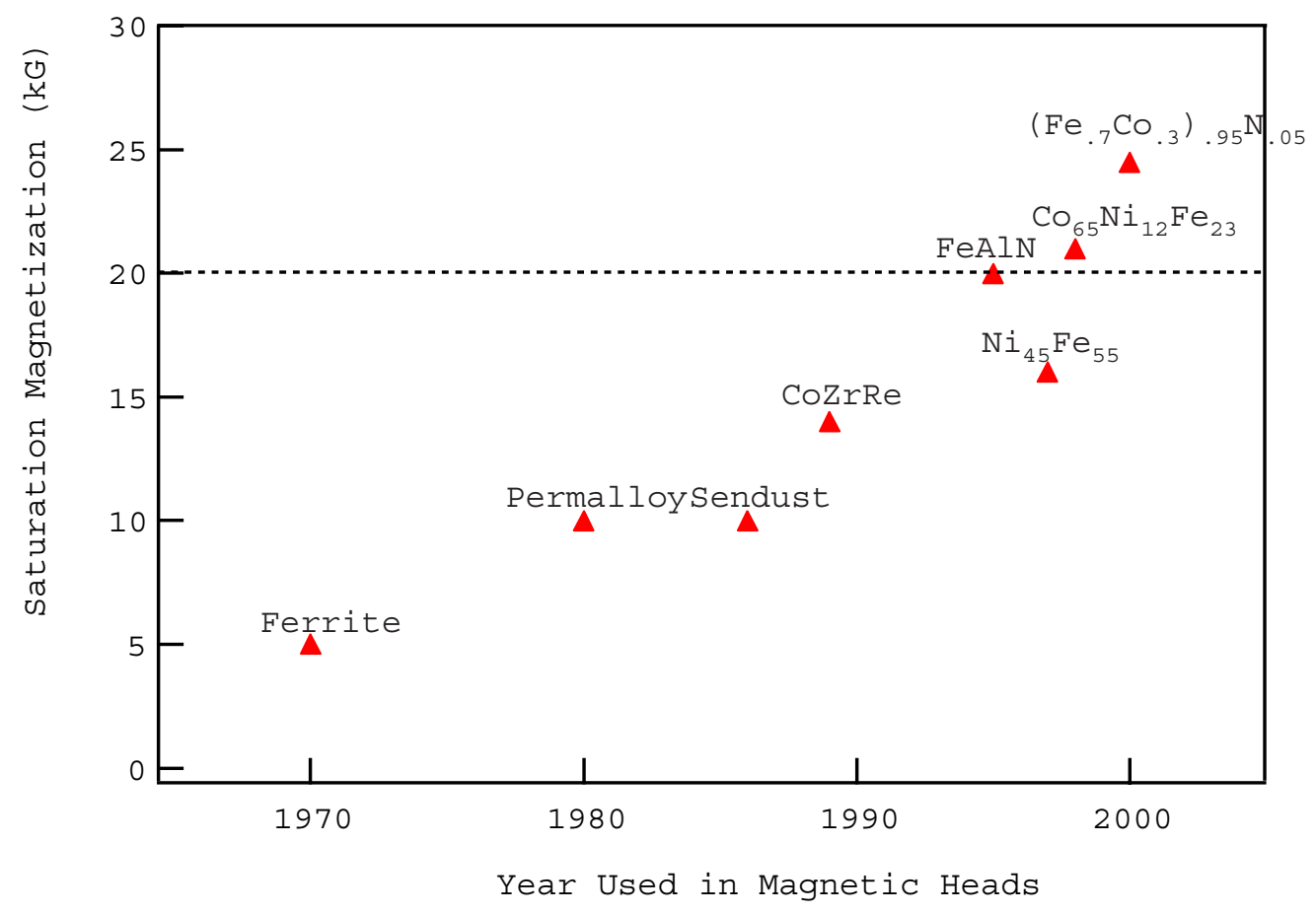

Figure 6: Saturation magnetization of soft magnetic materials for magnetic write heads, plotted against the year of first use in magnetic recording heads (except FeCoN). The dashed line indicates the perceived maximum saturation for magnetic write heads. This figure is meant to be representative rather than exhaustive.

\section{STRESS AND MAGNETOSTRICTION ANALYSIS}

The permalloy underlayer for the $\mathrm{P} \backslash \mathrm{FeCoN} \backslash \mathrm{P}$ and $\mathrm{P} \backslash \mathrm{FeCoN}$ structures might act as a buffer layer and relieve the stress of the whole film structure, this can be one of the reasons for the soft magnetic properties observed for the $\mathrm{P} \backslash \mathrm{FeCoN}$ and the $\mathrm{P} \backslash \mathrm{FeCoN} \backslash \mathrm{P}$ structures. Here, the stress values of the four film structures are measured by the wafer curvature method, and shown in Table 1. All the four film structures have almost identical compressive stress within measurement error, about -300 MPa. Therefore, variation in stress can be ruled out as a cause for the change in soft magnetism for the four film structures.

Since the FeCo materials are highly magnetostrictive, it is reasonable to deduce that the FeCoN materials might also have a very high saturation magnetostriction from the former work on the FeMN systems $[5,6]$. Therefore, we measured the saturation magnetostriction constants, $\lambda_{s, s}$, for the four films structures, and the measured results were also shown in Table 1 . The $\lambda_{s, s}$ is 
the average magnetostriction constant for the whole film structure by using the whole structure thickness during the measurement. We can see that all the saturation magnetostriction values are very high, just as the FeCo alloys [1]. However, we can still see a trend that the $\mathrm{P} \backslash \mathrm{FeCoN} \backslash \mathrm{P}$ structure has the lowest $\lambda_{s, s}$ value, and the $\mathrm{P} \backslash \mathrm{FeCoN}$ structure has the second lowest $\lambda_{s, s}$ value, although the differences are small. This trend in $\lambda_{s, s}$ coincides with the trend in coercivity.

Table 1: Measured stress $(\sigma)$, measured saturation magnetostriction constant for the whole structure $\left(\lambda_{s, s}\right)$, and calculated saturation magnetostriction constant for the FeCoN layer $\left(\lambda_{s, \mathrm{FeCoN}}\right)$, for the various film structures. Each stress value is an average of three measurements.

\begin{tabular}{ccccc}
\hline \hline Film structures & $\mathrm{FeCoN}$ & $\mathrm{FeCoN} / \mathrm{P}$ & $\mathrm{P} / \mathrm{FeCoN}$ & $\mathrm{P} / \mathrm{FeCoN} / \mathrm{P}$ \\
\hline \hline$\sigma(\mathrm{MPa})$ & $-307 \pm 40$ & $-300 \pm 40$ & $-322 \pm 40$ & $-337 \pm 40$ \\
\hline$\lambda_{s, s}\left(10^{-6}\right)$ & 44.3 & 43.9 & 41.4 & 39.6 \\
\hline$\lambda_{s, \mathrm{FeCoN}}\left(10^{-6}\right)$ & 44.3 & 46.1 & 43.5 & 43.6 \\
\hline \hline
\end{tabular}

It would be interesting to look at the saturation magnetostriction constants of the $\mathrm{FeCoN}$ layer in the four films structures. If we assume that: (a) film thickness is much thinner than the substrate; (b) $\varepsilon$ is the same for all layers in a film, then the total deflection angle $\phi$ for a multilayer film structure can expressed as:

$$
\phi=\frac{6 l \varepsilon}{t_{s}^{2}} \times\left(\sum_{i=1}^{n} t_{i} \lambda_{i}\right)
$$

where $t_{i}$ and $\lambda_{i}$ are the thickness and magnetostriction constants for the $i$ th layer of the whole film structure, and $n$ is the total number of layers for a whole film structure (for nonmagnetic layer, $\lambda_{i}$ $=0$ ). Combining equations 2 and 3 , we get:

$$
t_{s} \lambda_{s, s}=t_{\mathrm{p}} \lambda_{\mathrm{p}}+t_{\mathrm{FeCON}} \lambda_{\mathrm{FeCoN}}
$$

where $t_{s}$ is the total thickness of the film structure, $t_{\mathrm{p}}, \lambda_{\mathrm{p}}$ and $t_{\mathrm{FeCoN}}, \lambda_{\mathrm{FeCON}}$ are the thickness and magnetostriction constant for the permalloy layer and the FeCoN layer, respectively. Compared with the measured $\lambda_{s, s}$ values, the saturation magnetostriction constant for permalloy layer is very small $\left(<10^{-6}\right)$, and can be neglected. Therefore, by assuming $\lambda_{\mathrm{p}}=0$ for all the four film structures, we can calculate the saturation magnetostriction constant, $\lambda_{\mathrm{FeCoN}}$, for the FeCoN layer for all the film structures. As indicated in Table 1 , the $\lambda_{\mathrm{FeCON}}$ for the two film structures, $\mathrm{P} \backslash \mathrm{FeCoN} \backslash \mathrm{P}$ and $\mathrm{P} \backslash \mathrm{FeCoN}$, is almost the same. This is what we expected, as the FeCoN layer in the two film structures has the same permalloy underlayer, and the very thin permalloy caplayer would not change the FeCoN layer grain size much in the $\mathrm{P} \backslash \mathrm{FeCoN} \backslash \mathrm{P}$ film structure. While the higher calculated $\lambda_{\mathrm{FeCoN}}$ value in the FeCoN $\backslash \mathrm{P}$ film structure indicates that the permalloy caplayer layer may have a higher magnetostriction that the permalloy underlayers in the other film structures.

The coupling between the stress $\sigma$ and the magnetostriction strain $\lambda_{s}$ results in the magnetoelastic energy term $E_{m e}=3 / 2 * \lambda_{s} * \sigma$, which contributes to the total anisotropy of the 
polycrystalline magnetic thin films and can have significant effects on the magnetic softness. In the FeCoN films, however, the nearly identical stress and magnetostriction constants imply that the drastic magnetic softness difference observed in Fig. 5(a) and 5(d) does not result from the magnetoelastic effects.

\section{STRUCTURAL CHARACTERIZATION}

In the last section, dramatic changes have been observed in the soft magnetic properties of the FeCoN films after being sandwiched between two permalloy layers. In this section, we will analyze the grain size, film texture, coupling between the magnetostriction and the mechanical properties, trying to understand why these changes occur.

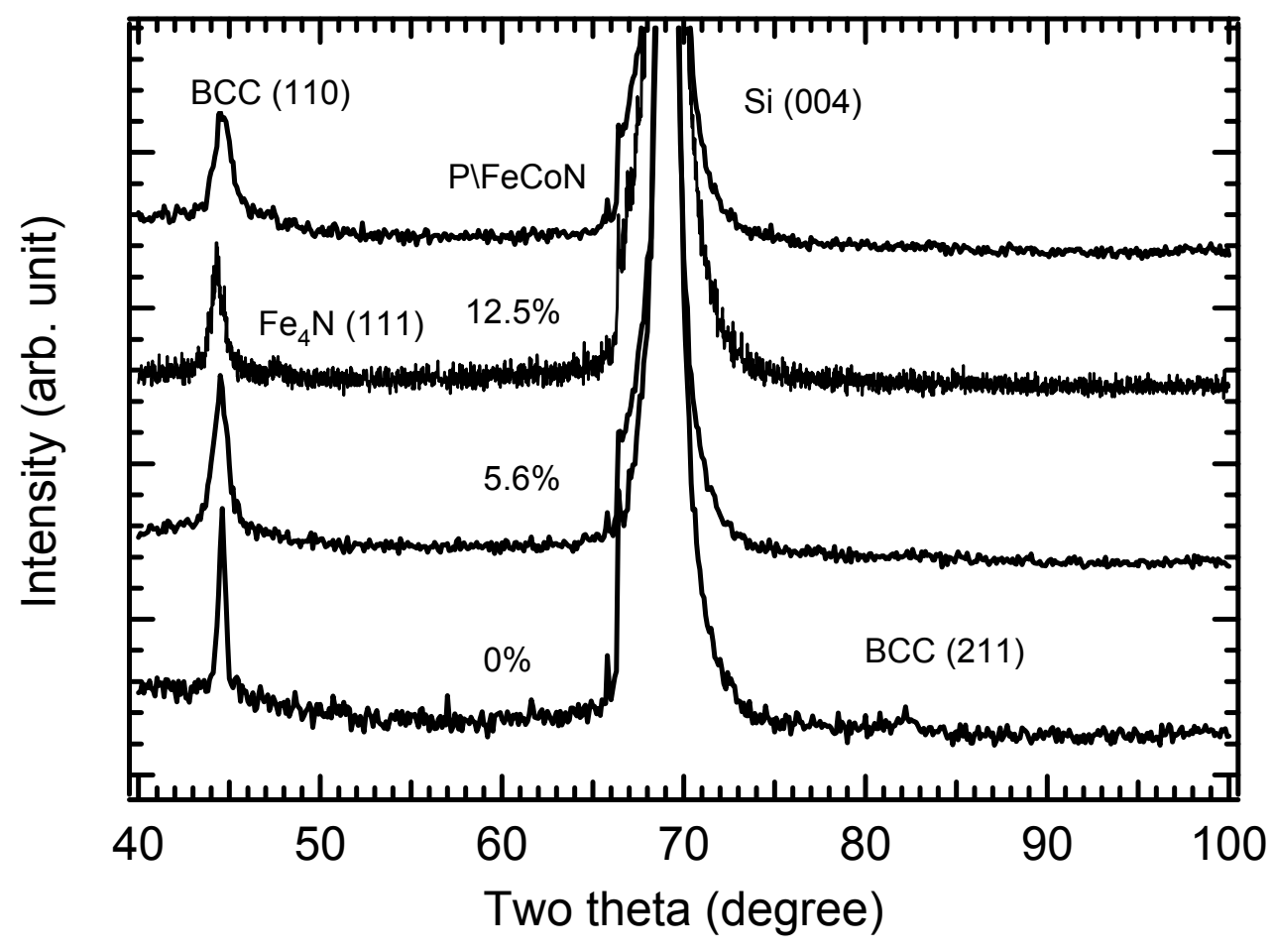

Figure 7: XRD patterns for the FeCoN single layers at different $\mathrm{N}_{2} /$ Ar gas flow rate ratios, $0 \%$, $5.6 \%$ and $12.5 \%$; and the XRD pattern of the P|FeCoN film structure as indicated in Figure 4.

Symmetrical $\theta-2 \theta$ XRD patterns of the FeCoN films deposited at three $\mathrm{N}_{2} /$ Ar gas flow rate ratios, $0 \%, 5.6 \%$, and $12.5 \%$ are shown in Fig. 7. The pure FeCo alloy film has a strong BCC $\alpha$ $\mathrm{Fe}(\mathrm{Co}, \mathrm{N})(110)$ and a weak BCC (211) Bragg peak; while at a $\mathrm{N}_{2} / \mathrm{Ar}$ gas flow rate ratios of $5.6 \%$, the FeCoN layer has a BCC structure with only a strong (110) diffraction peak, indicating a change of texture in the FeCoN films with a higher $\mathrm{N}$ content. At a $\mathrm{N}_{2} / \mathrm{Ar}$ gas flow rate ratios of $12.5 \%$, the $\mathrm{Fe}_{4} \mathrm{~N}$ phase appears. The $\mathrm{P} \backslash \mathrm{FeCoN}$ film shows a comparable XRD pattern to that of the FeCoN single layer with an identical $\mathrm{N}_{2} / \mathrm{Ar}$ gas flow rate ratios of $5.6 \%$. In addition, the $\alpha$ $\mathrm{Fe}(\mathrm{Co}, \mathrm{N})(110)$ diffraction peak is shifted to lower angles and is broader at a higher $\mathrm{N}_{2} / \mathrm{Ar}$ gas flow rate ratio. Shifting of the BCC (110) peak to lower angle implies that a higher $\mathrm{N}$ content is 
incorporated in the $\mathrm{FeCoN}$ films at a higher $\mathrm{N}_{2} / \mathrm{Ar}$ flow rate ratio; while a broader (110) peak indicates a smaller grain size and/or a higher micro-strain in the $\mathrm{FeCoN}$ films.

When the ferromagnetic exchange length is larger than the grain size, the magneto-crystalline anisotropy of the nanometer sized polycrystalline materials can be suppressed, which results in an improvement in magnetic softness [2]. Measurements of the full width at half maximum (FWHM) for the (110) diffraction peak in the symmetrical XRD patterns for the four film structures indicate the FWHM values are very close, indicating that the mean grain size for these four film structures may not be very much different. Therefore, grain size difference can not explain the different soft magnetic behaviors for the four film structures in Fig. 4.

Pole figure analysis provides a complete view of the 3-dimensional distribution of certain pole of the specimen. The $\mathrm{Fe}(\mathrm{Co}, \mathrm{N}) \mathrm{BCC}(110)$ pole figures for the $\mathrm{FeCoN}$ single layer and the FeCoN layer with the permalloy underlayer are shown in Figs. 8(a) and 8(b), respectively. The two pole figures all have the same (110) ring at $\chi=60^{\circ}$, corresponding to the strong (110) fiber texture for the $\mathrm{BCC} \mathrm{FeCoN}$ layer. In the pole figure of the $\mathrm{FeCoN}$ layer with a permalloy underlayer, there are two additional (110) diffraction rings at $\chi=30^{\circ}$ and $45^{\circ}$, respectively. These two rings corresponding to the (211) and (100) fiber texture, respectively, as the plane normal of (211) and (100) are $30^{\circ}$ and $45^{\circ}$ apart from that of (110), respectively. Changes in pole figures can bring changes in material properties for the highly anisotropic $\mathrm{BCC}$ FeCoN material, such as the distribution of magnetic easy axis of the individual crystals, the biaxial modulus, the magnetostriction constants, etc., eventually leading to the changes to the soft magnetic properties of thin films [3]. However, more work need to be done to clarify this because of the complexity of the pole figures observed.

\section{CONCLUSIONS}

We successfully deposited soft $\mathrm{FeCoN}$ single layers with a high saturation magnetization of $24.5 \mathrm{kG}$ and a coercivity of 5 Oe. A low coercivity of $0.6 \mathrm{Oe}$, and an excellent in-plane uniaxial anisotropy with an anisotropy field of 20 Oe were achieved in the FeCoN films sandwiched between two very thin Permalloy layers. This film structure has an initial permeability of 1000

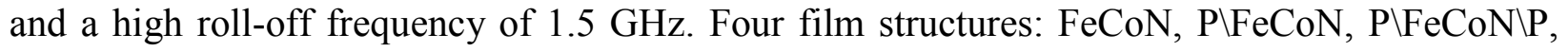
and $\mathrm{FeCoN} \backslash \mathrm{P}$, have been fabricated and their magnetic and structural properties compared in order to determine why soft magnetic properties are achieved in the FeCoN layer sandwiched between two permalloy layers. The stresses for the different film structures are $-300 \mathrm{MPa}$, almost the same for the four film structures; and the saturation magnetostriction constants are only slightly different for different film structures. There are differences in the fiber texture of the FeCoN single layer and the $\mathrm{P} \backslash \mathrm{FeCoN}$ film structure. 

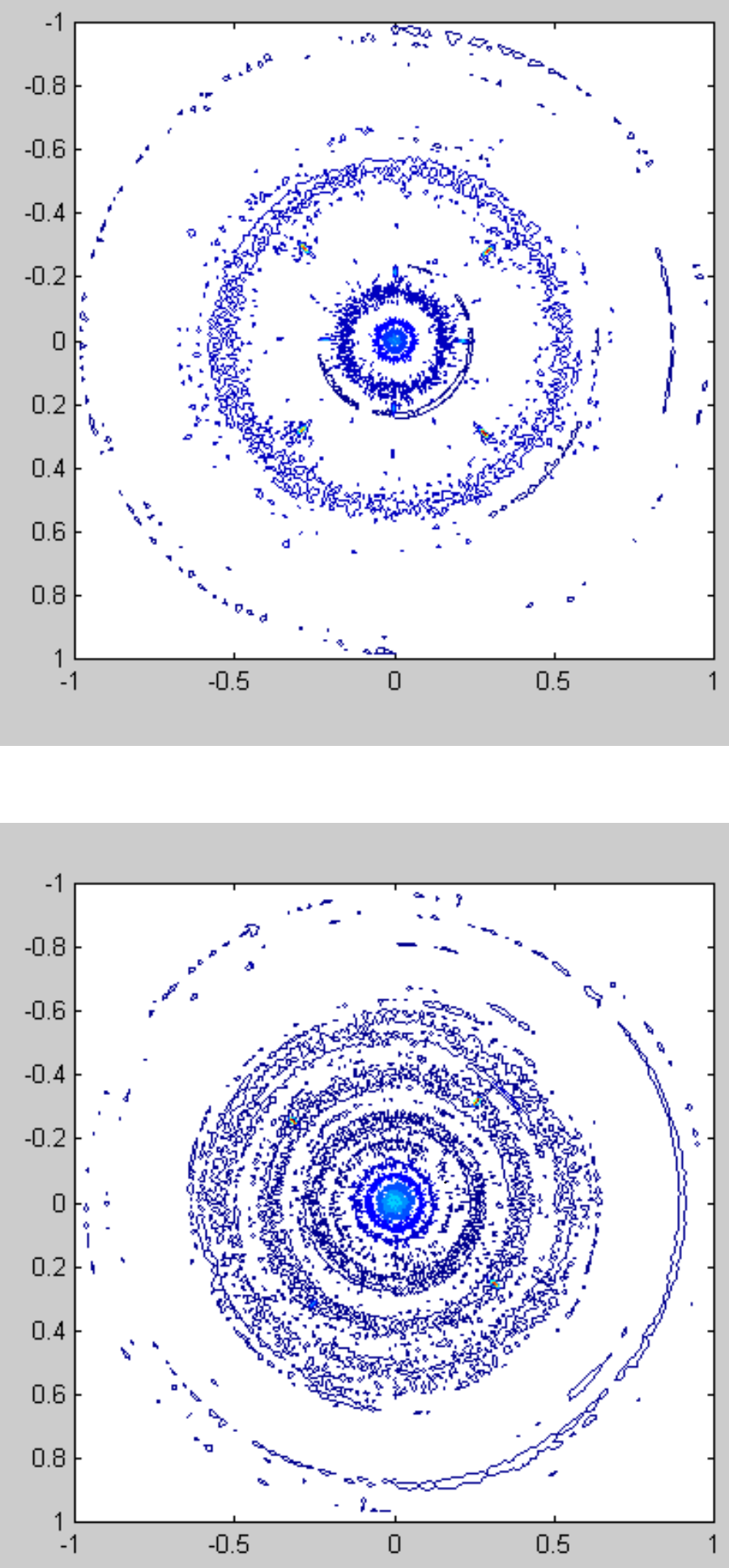

Figure 8 Typical Pole figure for the BCC FeCoN (110) diffration of the FeCoN structure (up); and the P|FeCoN structure (down). 


\section{ACKNOWLEDGEMENTS}

We are grateful to the helpful discussion with professor W. D. Nix and Dr. Omar Leung (Stanford University) on the stress measurement. Dr. Ann Marshall (Stanford University) helped us on the HRTEM observation. Dr. M. Yamaguchi and Dr. S. Yabukami at the Research Institute of Electrical Communication at Tohoku University are acknowledged for their help on the high frequency permeability measurements. This work is supported in part by NSF under grant ECS9710223.

\section{REFERENCE}

1. Richard M. Borzorth, Ferromagnetism, (IEEE press, 1993).

2. G. Herzer, J. Appl. Phys., 26, 1397 (1990).

3. Akihiko Hosono and Y. Shimada, J. Appl. Phys., 67, 6981 (1990).

4. B. D. Cullity, Introduction to magnetic materials, (Addison-Wesley, 1972).

5. M. H. Kryder, S. X. Wang, and K. Rook, J. Appl. Phys. 73, 6212 (1993).

6. B. Viala, M. K. Minor, and J. A. Bernard, J. Appl. Phys. 80, 3941 (1996).

7. S. Jin, W. Zhu, T. H. Tiefel, V. Korenivski, and R. B. van Dover, L. H. Chen, J. Appl. Phys. 81, 4042 (1997).

8. H. Ono, M. Ishida, M. Fujinaga, H. Shishido, and H. Inaba, J. Appl. Phys. 74, 5124 (1993).

9. E. J. Yun, W. Win, and R. M., Walser, IEEE Trans. Mag., 32, 4535 (1996).

10. P. C. Kuo, S. S. Chang, and C. M. Kuo, Y. D. Yao, and H. L. Huang, J. Appl. Phys. 83, 6643 (1998).

11. S. Yabukami, M. Yamaguchi, K. I. Arai, M. Watanabe, A. Itagaki, H. Ando, J. Appl. Phys., 85, 5148 (1999).

12. W. D. Nix, Metall. Trans. A, 20A, 2217 (1989).

13. A. C. Tam and H. Schroeder, IEEE Trans. Magn., 25, 2629 (1989).

14. H. Clow, Nature, 194, 1035 (1962).

15. S. X. Wang, N. X. Sun, M. Yamaguchi and S. Yabukami, (in print), Nature (2000). 